\title{
BOUNDS FOR THE RATIOS OF DIFFERENCES OF POWER MEANS IN TWO ARGUMENTS
}

\section{OMRAN KOUBA}

Abstract. Using methods from classical analysis, sharp bounds for the ratio of differences of power means are obtained. Our results generalize and extend previous ones due to $\mathrm{S}$. $\mathrm{Wu}(2005)$, and to S. Wu and L. Debnath (2011).

Mathematics subject classification (2010): 26E60, 26D07.

Keywords and phrases: Arithmetic mean, geometric mean, power mean, L'Hospital Monotone Rule.

\section{REFERENCES}

[1] H. AlzER AND S.-L. QIU, Inequalities for means in two variables, Archiv der Mathematik (Basel), 80, no. 2, (2003), 201-215.

[2] G. Anderson, M. Vamanamurthy and M. Vuorinen, Monotonicity Rules in Calculus, American Mathematical Monthly, 113, no. 9, (2006), 805-816.

[3] J. CheEger, M. Gromov AND M. TAYLOR, Finite propagation speed, kernel estimates for functions of the Laplace operator, and the geometry of complete Riemannian manifolds, Journal of Differential Geomety 17, no. 1, (1982), 15-53.

[4] O. KoubA, New bounds for the identric mean of two arguments, Journal of Inequalities in Pure and Applied Mathematics, 9, no. 3, (2008) Art. 71, Available at http://www.emis.de/journals/ JIPAM/article1008.html.

[5] T. TRIF, Note on certain inequalities for means in two variables, Journal of Inequalities in Pure and Applied Mathematics, 6, no. 2, (2005) Art. 43, Available at http://www.emis.de/journals/ JIPAM/article512.html.

[6] S. WU, Generalization and sharpness of power means inequality and their applications, Journal of Inequalities in Pure and Applied Mathematics, 312, no. 2, (2005), 637-652.

[7] S. Wu And L. Debnath, Inequalities for differences of Power Means in two variables, Analysis Mathematica, 37, no. 2, (2011), 151-159. [DOI:10.1007/s10476-011-0203-z] 